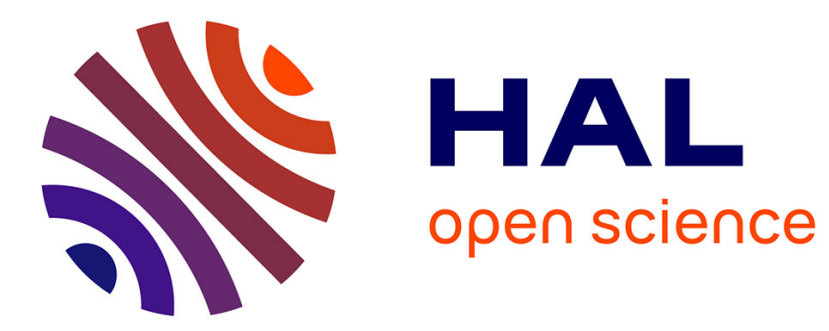

\title{
Le cothon ou port artificiel creusé. Essai de définition \\ Nicolas Carayon
}

\section{To cite this version:}

Nicolas Carayon. Le cothon ou port artificiel creusé. Essai de définition. Méditerranée: revue géographique des pays méditerranéens, 2005, 1 (2), pp.5-13. halshs-00115127

\section{HAL Id: halshs-00115127 https://shs.hal.science/halshs-00115127}

Submitted on 20 Nov 2006

HAL is a multi-disciplinary open access archive for the deposit and dissemination of scientific research documents, whether they are published or not. The documents may come from teaching and research institutions in France or abroad, or from public or private research centers.
L'archive ouverte pluridisciplinaire HAL, est destinée au dépôt et à la diffusion de documents scientifiques de niveau recherche, publiés ou non, émanant des établissements d'enseignement et de recherche français ou étrangers, des laboratoires publics ou privés. 
Article paru dans « Méditerranée » N¹.2 2005 


\title{
I - TECHNOLOGIES, HÉRITAGES ET MOBILITÉS À L'ÉCHELLE DES BASSINS PORTUAIRES
}

\section{Le cothon ou port artificiel creusé. Essai de définition}

\author{
Defining the cothon or artificially dug harbour
}

Nicolas CARAYON*

\begin{abstract}
Résumé - Les sites archéologiques de Carthage, Mahdia, Phalasarna, Motvé, Rachgoun et Jezirat Fara'un ont en commun la présence d'un port appelé "cothon». Ce terme est utilisé depuis l'Antiquité pour désigner le port de Carthage et, aujourd'hui, les spécialistes d'archéologie portuaire s'accordent pour l'associer à un "bassin portuaire creusé et débouchant sur la mer libre par l'intermédiaire d'un chenal». Ce travail, après un bref inventaire des sources antiques et archéologiques, se propose de revenir sur la définition archéologique du terme, sur la vocation d'un tel type de port, et sur l'origine, peut-être orientale, d'une telle technique d'aménagement portuaire.
\end{abstract}

Les archéologues s'accordent à définir un cothon comme un bassin portuaire creusé et débouchant sur la mer libre par l'intermédiaire d'un chenal (HADJIDAKI, 1988; Debergh et Lipinski, 1992 ; Frost, 1995). Cette définition, qui se base sur différents auteurs antiques, a permis d'associer le terme de cothon à six ports antiques (Carthage, Mahdia, Phalasarna, Motyé, Rachgoun et Jezirat Fara'un) qui, quelquefois, n'ont jamais été nommés ainsi dans l'Antiquité (fig. 1). Les progrès relativement récents de l'archéologie portuaire justifient une mise au point sur l'utilisation du terme. Ce travail de synthèse se donne pour objectif, en s'appuyant sur les dossiers philologiques et archéologiques, de proposer une nouvelle définition archéologique du cothon, en insistant sur la technique mise en œuvre, sa diversité et son origine.

\section{Les sources antiques}

Chez les auteurs gréco-romains, le terme de cothon peut avoir, en plus d'une utilisation attestée en tant que nom propre, deux significations différentes. Chez certains auteurs grecs (Athen. XI ; Plutarque, Lycurg., 9 ; Pollux,
Abstract - The archaeological sites of Carthage, Mahdia, Phalasarna, Motyé, Rachgoun and Jezirat Fara'un share in common the presence of a port called «cothon». This term is used since antiquity to refer to the port of Carthage. Nowadays, the specialists of harbour archaeology agree that the term can be associated to dug harbour-basin emerging on the sea through a channel. This work, after a short inventory of ancient and archaeological sources, is made in order to reanalyse the definition of the term under study, the vocation of that such kind of harbour, and the origin of that technique of harbour planning, which is possibly an oriental one.

Onomasticon, VI, 16 ; Xénophon, Cyropédie, I, 2, 8), un cothon $(K \omega \theta \omega v)$ est un vase à boire d'origine lacédémonienne. Ce vase rond à anse unique, avait un rebord épais à l'embouchure et un col rentré à l'intérieur (fig. 1). Il pouvait être en argile et utilisé spécialement par les marins et les soldats, ou en bronze et placé comme offrande dans les temples (ViedebantT, 1922 ; DAREMBERG et SAGLio, 1962-63).

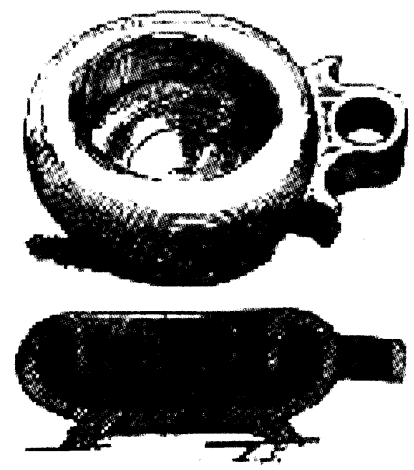

FIG. 1 - UN COTHON (Daremberg et Saglio, 1962)

* Université Marc Bloch, Palais Universitaire - 9, place de l'Université- 67084 Strasbourg Cedex ; UMR 7044 «Étude des civilisations de l'Antiquité». CNRS. 


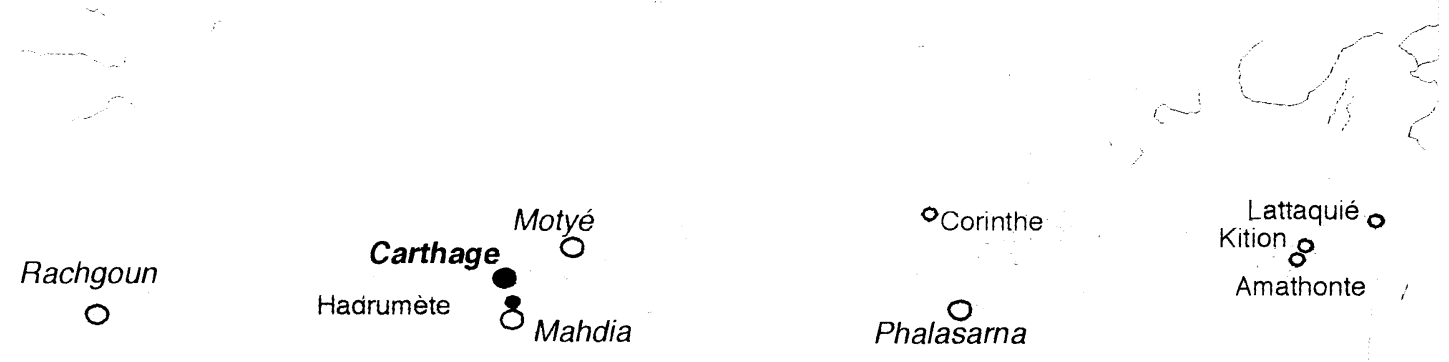

- Cothon attesté dans les sources antiques et par l'archéologie

- Cothons attestés par l'archéologie

- Cothon attesté dans les sources antiques

- Cothons possibles

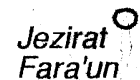

II(i. 2 - (ARTI I)I RIAPARTITION I)ISS COTHONS

La deuxième signification du terme, celle qui attirera toute notre attention, est celle qui en fait une technique d’aménagement portuaire. Les sources les plus anciennes, autour du changement d'ère, associent systématiquement le terme de cothon au(x) fameux port(s) de Carthage ou à celui d'Hadrumète (l'actuelle Sousse en Tunisie). Chez. Appien (Lib. 127), le $K(\hat{\omega} \theta \omega v$ semble se rapporter aux deux bassins, rectangulaire et circulaire. de Carthage. En revanche, che' Strabon (XVII, 3, 14), la mention des hangars à navires en périphérie du bassin semble se rattacher uniquement au port circulaire. Quant à Diodore de Sicile ( $\mathrm{I}^{\mathrm{c}} \mathrm{s}$ s. av. J.-C.) il indique simplement que le port de Carthage était connu sous le nom de $K\left(\dot{s} \theta \omega \omega^{\prime}\right.$ (III, 44, 7-8).

A Hadrumète, l'existence d'un cothon est mentionnée à trois reprises dans le Bellum Africum $(62$. $5 ; 63,4 ; 63,5)$, attribué à César. Ce port était le reluge des navires militaires de Varus. Nous noterons que A. Bouvet, l'auteur de la traduction dans l'édition des Belles Lettres (1949) traduit cothon par «port intérieur».

Les sources antiques visant à définir le port de type cothon semblent en partie reposer sur un passage de Virgile (71 av.-19 ap. J.-C.) relatif aux ports de la Carthage augustéenne : «Hic portus alii effodiunt» (Aen., I 427) : le cothon de Carthage est alors un port creusé. Deux comnentateurs de l'Enéide de Virgile, ont proposé une définition du terme appliqué aux ports de Carthage. Le premier Servius écrit : "Cothona sunt portus in mari, non naturales, sed arte' et manu facti» (in Verg. Aen., I 427). Il insiste sur les caractères artificiel et maritime du cothon, sans aucune précision sur la technique par laquelle il fut élaboré. Un deuxième commentateur de Virgile, le Déutero-Servius, nous donne la définition suivante: "Portus effodiunt, ut portus scilicet faciunt. Et vere ait. nam Carthaginienses Cothone fossa utuntur, non naturali portus's. On retrouve ici le caractère artificiel du cothon. ainsi que la technique du creusement mentionnée par Virgile. Il est intéressant de noter que le Deutero-Servius sous-entend que le creusement des ports était une technique courante.

Deux autres auteurs latins nous proposent une définition du cothon. il sagit de deux grammairiens latins.
Sextus Pompeius Festus et Lactantius Placidus. Selon Festus : "Cothoness appellantur portus in mari interiores arte at manu facti» (De verborum significatu, III). Lauteur insiste ici encore sur les côtés maritime et artiliciel du cothon. De plus le qualificatif interiores, qui se rapporte à portus et non pas à mari, pourrait faire allusion à un port situé à l'intérieur de l'enceinte, ce qui est largement décrit par Appien à Carthage (Lib. 96). Finalement Lactantius Placidus (Glossae V, 19, 13) mentionne: "Coton cubiculum graece кoutóv est. (cotonem) ergo quod Carthago habet, in quo naves ('lauduntur; recte cubiculum dicimus'». L'auteur préfère au terme de port, le mot (ubiculum (chambre) que nous tendrons à lire «bassin». Il mentionne également un équivalent grec : кoıt définition est la référence qu'elle fait au port de Carthage.

Quant à l'origine étymologique du terme, deux écoles s'opposent. La première, «sémitisante», rattache le terme cothon à une racine sémitique que l'on retrouverait dans l'arabe quttar (tailler, couper), et qui s'accorderait avec le sens de port. Cette racine n'est pourtant pas attestée dans les textes sémitiques anciens et le seul rapprochement possible étant avec la racine qtn (petit), que l'on retrouve en akkadien (qutanu: parcelle) (Debergh et Lipinski, 1992). La seconde école, "hellénisante», donnerait au terme une origine grecque (HADJIDAKI, 1988). Si cela peut être le cas en ce qui concerne le nom d'une céramique, le seul indice qui en ferait un port est une similitude de forme entre le vase cothon et le port circulaire de Carthage.

Si les auteurs antiques identifient comme cothon les ports de Carthage et d'Hadrumète, seul celui de Carthage nous permet une étude archéologique. À Hadrumète, les travaux archéologiques n’ont jamais pu mettre au jour les vestiges d'un tel aménagement (Folchir. 1964). Cependant, la présence des vestiges archéologiques permet d’associer le terme de cothon aux vestiges de six ports antiques. Ces sites sont (fïg. 2) : Carthage et Mahdia en Tunisie, Phalasarna sur la côte occidentale de la Crète, l'île de Motyé en Sicile, et l'île de Rachgoun en Algérie. Jezirat Fara un. également une île. 
se trouve en mer Rouge. en Israël, au fond du golfe d’Aqaba. Tous ces établissements ont en commun la présence du noyau constitutif d'un cothon : un bassin et un chenal qui lui donne un accès à la mer libre. Chacun présente cependant des caractères qui lui sont propres. autant par leur nature que dans leur conception.

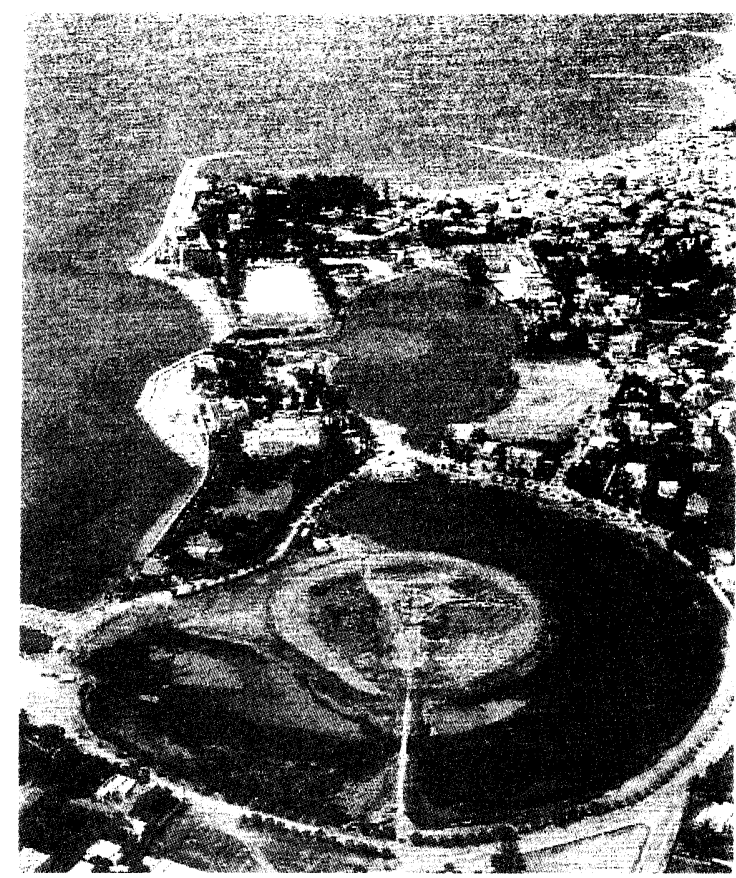

PHOTO 1 - VUE AÉRIENNE DES PORTS DE CARTHAGE (HURST, 1992)

\section{Les sources archéologiques}

Le vaste complexe de Carthage (fig. 3 et photo 1) est composé par un chenal débouchant sur la mer libre, sans doute au niveau du port extérieur, mais dont le tracé reste à préciser : d'un bassin rectangulaire, également appelé «port marchand»; et d'un bassin circulaire, le «port militaire». Le chenal reliant le bassin rectangulaire à la mer libre, au niveau du quadrilatère de Falbe, est aujourd'hui comblé. Une étendue d'eau visible dans les années 1930 pourrait en avoir constitué un vestige (BARADEZ, 1958). Le port rectangulaire est long de $400 \mathrm{~m}$ et large de $150 \mathrm{~m}$, sa profondeur dans l'Antiquité a été estimée à $2 \mathrm{~m}$ (Hurst et Stager, 1978). Les fouilles de la berge ouest ont mis au jour un quai construit de gros blocs $(2,3 \times 2,2 \times 0,75 \mathrm{~m})$ en grès d'El Haouaria, les assises les plus basses ont été appareillées sans liant. Le creusement de ce bassin a été daté de la fïn du IV"-III's. av. J.-C. (STAGer, 1992). Quoi qu'il en soit, son antériorité sur le port «militaire» ne fait aucun doute. En effet, le port circulaire $(325 \mathrm{~m}$ de diamètre et profond de $2 \mathrm{~m}$ ) fut creusé au milieu du II" s. av. J.-C. (Hurst et Stager, $1978:$ Hurst, 1993). Il

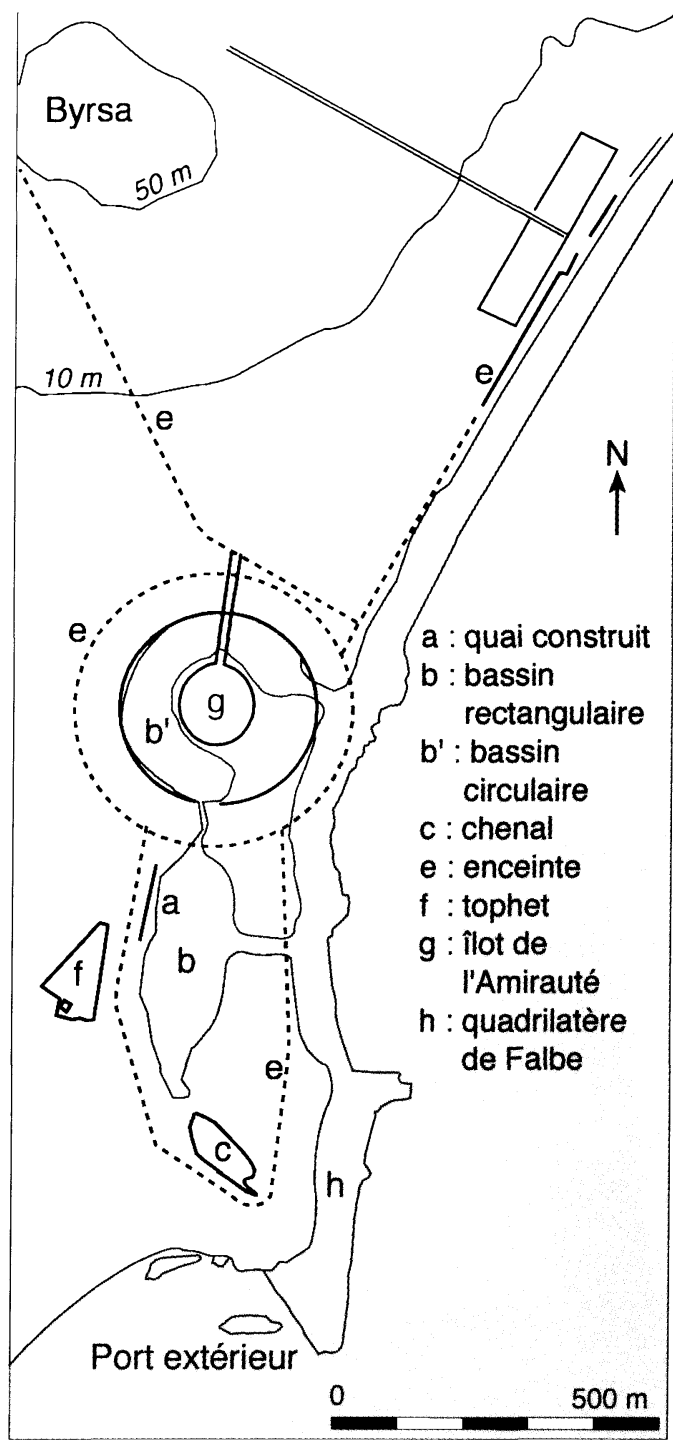

FIG. 3 - LES PORTS PUNIQUES DE CARTHAGE (d'après HURST, 1993)

comporte un îlot central de $125 \mathrm{~m}$ de diamètre, l'îlot de l'Amirauté (fig. 4) d'après le récit d'Appien (Lib., 96), qui donne au plan d'eau l'aspect d'un anneau large de $100 \mathrm{~m}$. Cet îlot était traversé au nord par une passerelle joignant l'îlot au rivage. La totalité des berges ont accueilli quelques 180 hangars à navires (30 sur l'îlot et 140 en périphérie du bassin). L'extrémité extérieure des rampes

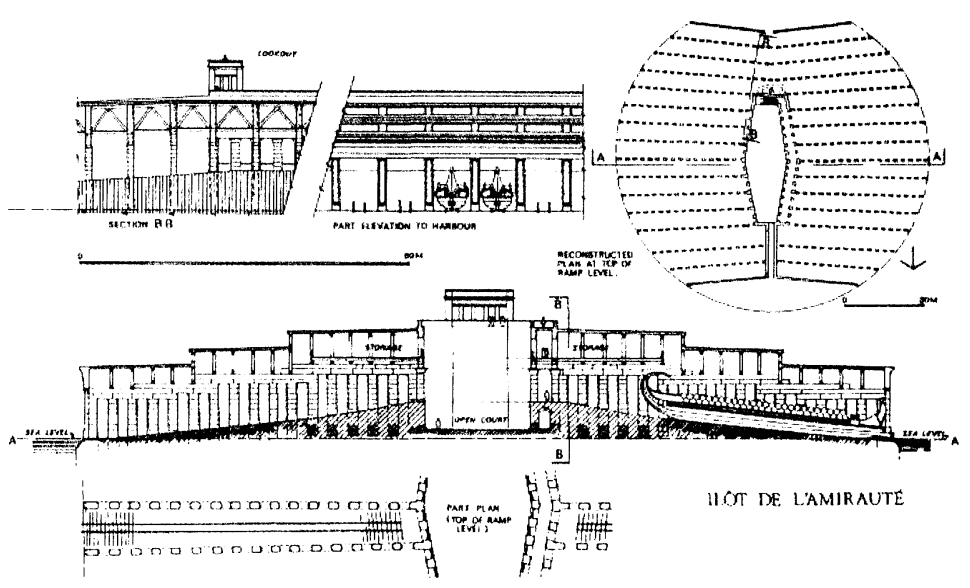

FIG. 4 - LE PORT CIRCULAIRE DE CARTHAGE

Restitution des hangars à navires sur l'îlot de l'Amirauté ((Hurst, 1993) 


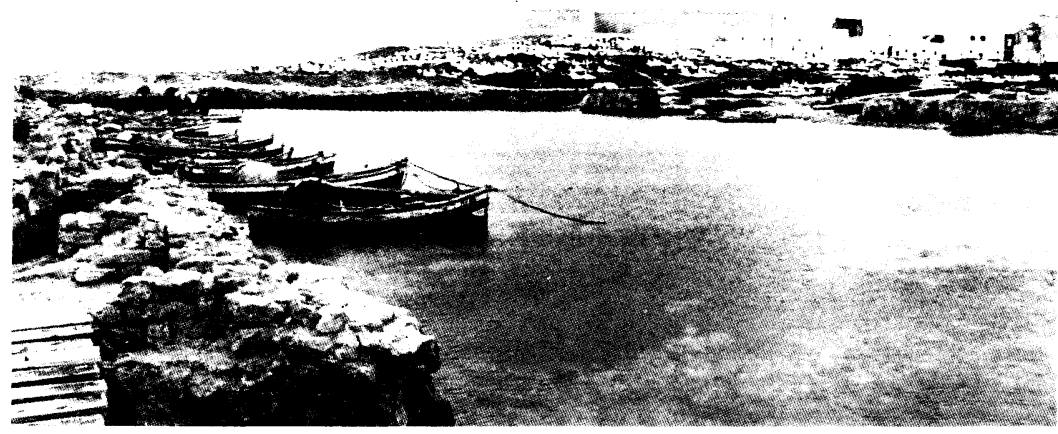

PHOTO 2 - LE COTHON DEE MAHIDIA, VU DEPUIS L ANGLE EST

PHOTO 3 - BITTE D`AMARRAGiE TAILLÉE À MAHDIA

(Clichés: CARAYON, 2()0)3)

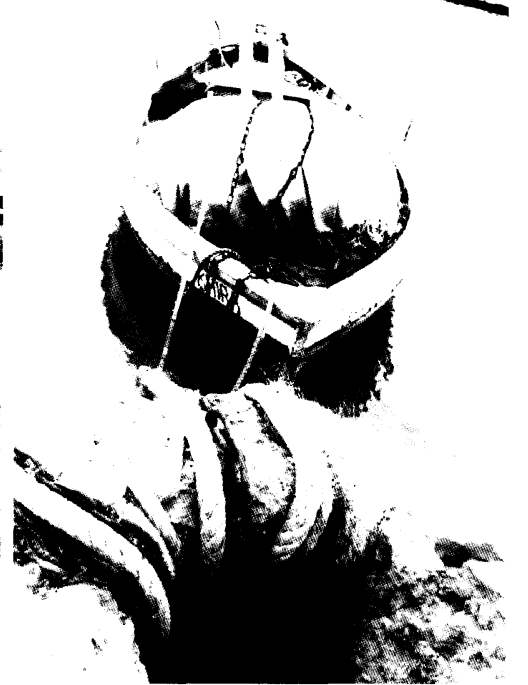

barques de pêcheurs viennent, aujourd'hui encore, s'échouer sur la section de grève ou s'amarrer aux quais. Des ergots rocheux traversés verticalement par un trou font office d'amarrage. La datation du cothon de Mahdia n'est pas encore fermement établie. Le creusement, du bassin et du chenal, est attribué soit au calife fatimide Ubaydullah, qui fut à l'origine, au $x^{c} \mathrm{~s}$. de notre ère, de la construction à Mahdia d'une important arsenal, soit aux Carthaginois (Lezine, 1965 ; Zaouali, 1999 ; Fantar, 1999). À l'extrémité est du promontoire, seuls quelques caveaux funéraires érodés par la mer pourraient être de facture punique. En revanche, une nécropole dont quelque 10(0) tombes ont été fouillées, s'étendait sur $12 \mathrm{~km}$ le long du littoral au nord du promontoire (BEN YOUNES, 1985). La date supposée pour les débuts de l'utilisation de cette nécropole, peut-être dès la fin du vis. av. J.-C. (FANTAR, 1999), plaiderait en faveur d'un établissement carthaginois à proximité du port creusé. Cette hypothèse est confortée par la présence, sur les parois du bassin, d'une encoche d'érosion marine, dont l'importance (hauteur $60 \mathrm{~cm}$, profondeur 0,4 à $1,1 \mathrm{~m}$ ) n'a jamais été atteinte en Tunisie sur des sites plus tardifs (PASkofF, SLim et Trousset, 1991 ; OuesLati, 1993). Il est done probable que le bassin fut taillé à l'époque punique.

Au début de l'époque hellénistique, à l'extrême fin du IV s. av. J.-C., le complexe portuaire de Phalasarna fut aménagé (fig. 6). Il est constitué par au moins un bassin très grossièrement rectangulaire $(100 \times 75 \mathrm{~m})$, relié à la mer par un chenal long d'une centaine de mètres et large de 10-12 m. Les mesures effectuées au sein du bassin et à sa jonction avec le chenal, restituent une profondeur antique comprise entre $1.1 \mathrm{~m}$ et $2 \mathrm{~m}$ (Pirazzoli et al., 1982 ; HADJIDAKI, $1988 ; 1996$; Frost, 1997). Au sud du bassin, un quai fut construit avec des blocs de calcaire $(1,1 \times 0,6 \times 0,4 \mathrm{~m})$ disposés en boutisses. Un chenal secondaire, divergent du chenal principal, était probablement destiné à lutter contre l'ensablement (id.). On a supposé l'existence d'un autre bassin portuaire, de $50 \mathrm{~m}$ de long et $35 \mathrm{~m}$ de large, situé plus au nord et relié au premier par un étroit chenal ; elle paraît peu probable. Sans doute. comme il a été suggéré, doit-on considérer ce bassin comme destiné à contenir de l'eau douce (id.). 


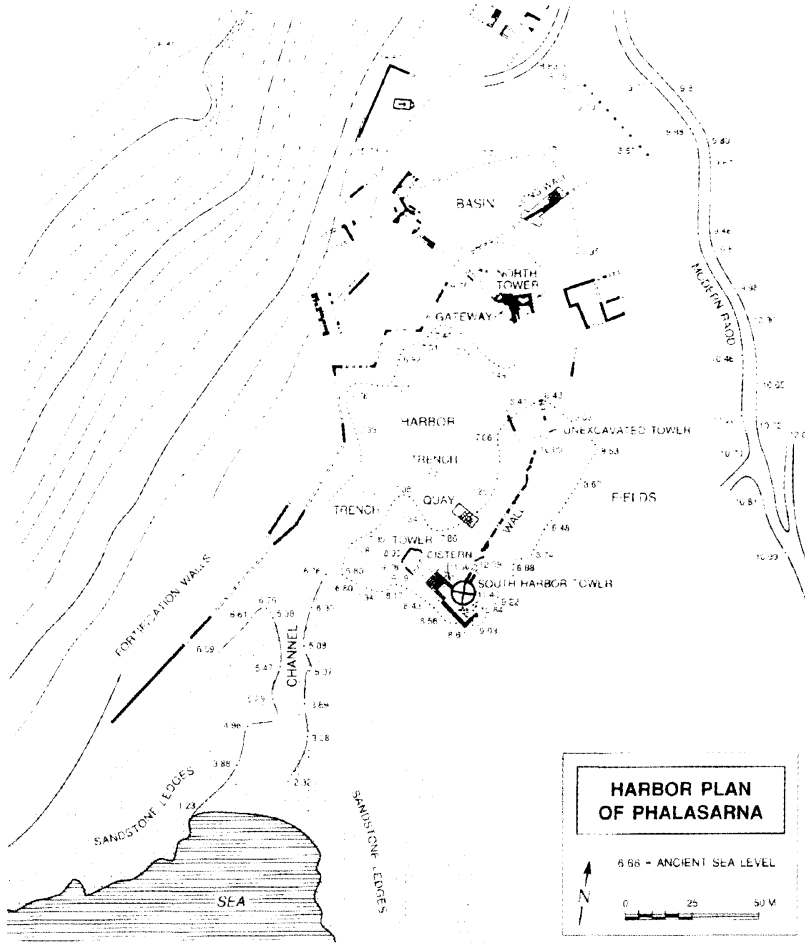

FIG. 6 - LE PORT DE PHALASARNA (Frost, 1997)

À Motyé (fig. 7, photo 4 et 5), le cothon, établi dans la deuxième moitié du $\mathrm{VI}^{\circ}$ s. av. J.-C., est constitué par un chenal long d'environ $30 \mathrm{~m}$ et d'un bassin rectangulaire, long aujourd'hui de $51 \mathrm{~m}$ et large de $35,5 \mathrm{~m}$ (DU PLAT TAYLOR, 1964 ; ISSERLIN, 1971 et 1974). Le chenal se divise en trois parties distinctes, la partie centrale ayant fait l'objet d'un soin particulier. Large de $5,8 \mathrm{~m}$ et profonde de $1,7 \mathrm{~m}$, ses berges sont construites de gros blocs de calcaire, disposés en boutisses ; l'assise inférieure de ce mur est marquée par un emmarchement. Le fond est constitué d'un pavement en blocs de taille variable avec, dans l'axe longitudinal, une rainure de section courbe, large de $54 \mathrm{~cm}$ et profonde de $13 \mathrm{~cm}$ (ISSERLIN, 1971 et 1974). Les sections nord et sud du chenal sont légèrement désaxées par rapport à la section centrale. Celle au sud, large de $7 \mathrm{~m}$, est trop incomplète pour que l'on puisse y observer un quelconque aménagement des berges. La section nord avait la forme d'un entonnoir, s'élargissant à sa jonction avec le bassin

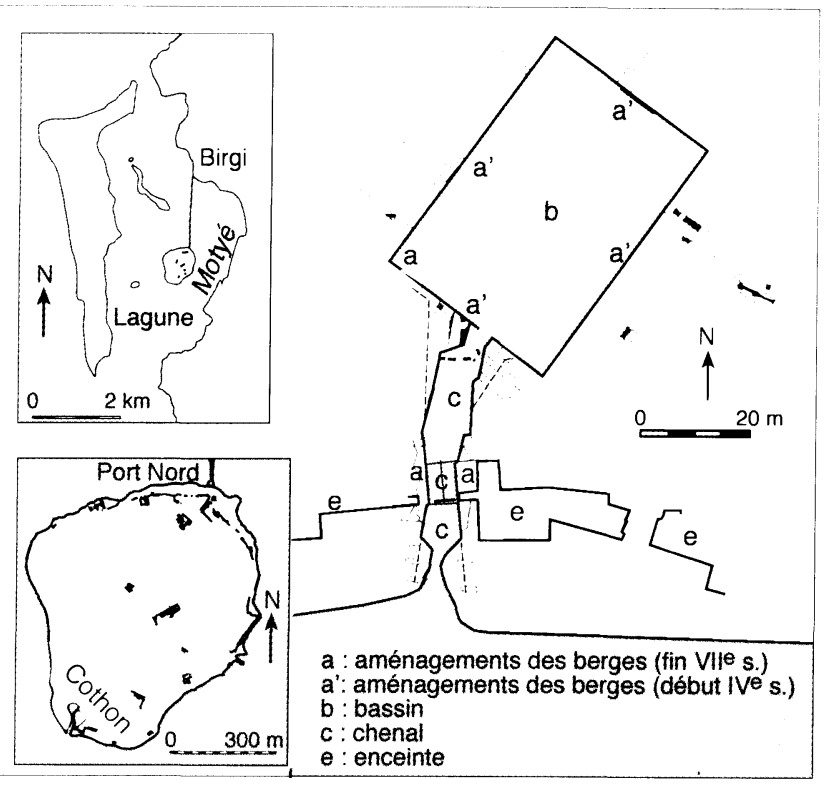

FIG. 7 - L'ÎLE DE MOTYÉ ET SON COTHON

(d'après ISSLERL.IN, 1974)

jusqu'à atteindre $17 \mathrm{~m}$. Il semble que ses berges furent d'abord taillées en pente puis, peu à peu, construites de manière à donner à tous les points du chenal la même largeur. À cette époque (fïn VII" s. -début VI"s. av. J.-C.) le bassin était plus étendu vers le nord qu'actuellement et ses berges n'étaient pas construites sur toute sa périphérie. Seule une section de mur dans l'angle sud-ouest du bassin pourrait dater de ce premier état (id.). Certainement très peu de temps avant la chute de la ville en 396 av. J.-C., les habitants de Motyé modifièrent considérablement la configuration du cothon : un mur de carreaux et boutisses fut élevé tout autour du bassin ; du côté nord, ce mur, légèrement incurvé, présente en son centre un rebord de $30 \mathrm{~cm}$ de large et 7,8 $\mathrm{m}$ de long. Finalement le chenal fut fermé par un mur situé au sud de la section pavée, entre les contreforts (id.). L'interprétation du bassin comme port ne semble faire aucun doute pour le premier état du complexe, lorsqu'il était relié à la mer par le chenal. En revanche, les modifications intervenues vers l'extrême fin du $V^{i} s$. ou le tout début du IV $V^{\mathrm{s}} \mathrm{s}$. pourraient en avoir fait un bassin sans lien direct avec la mer, lui enlevant par là toute vocation portuaire (MINGAZZINI, 1968).
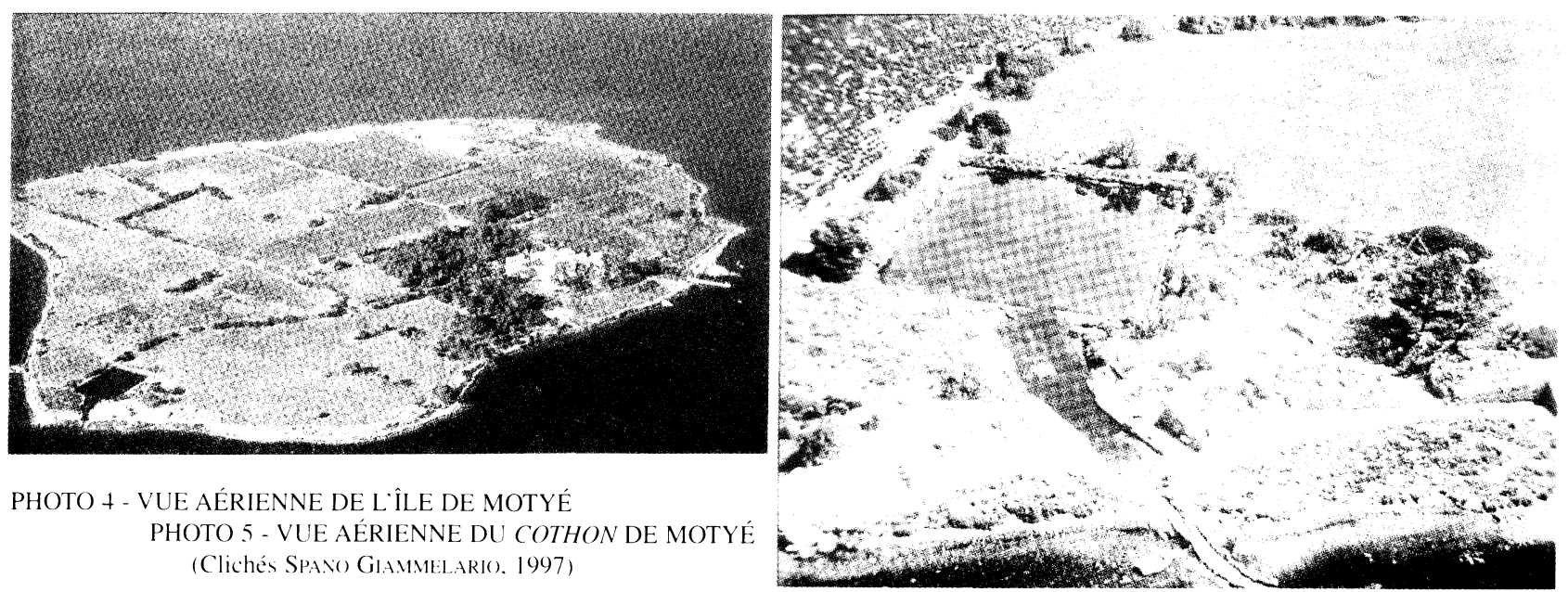

PHOTO + - VUE AÉRIENNE DE L ÎLE DE MOTYÉ PHOTO 5 - VUE AÉRIENNE DU COTHON DE MOTYE (Clichés Spano Giammelario), 1997) 


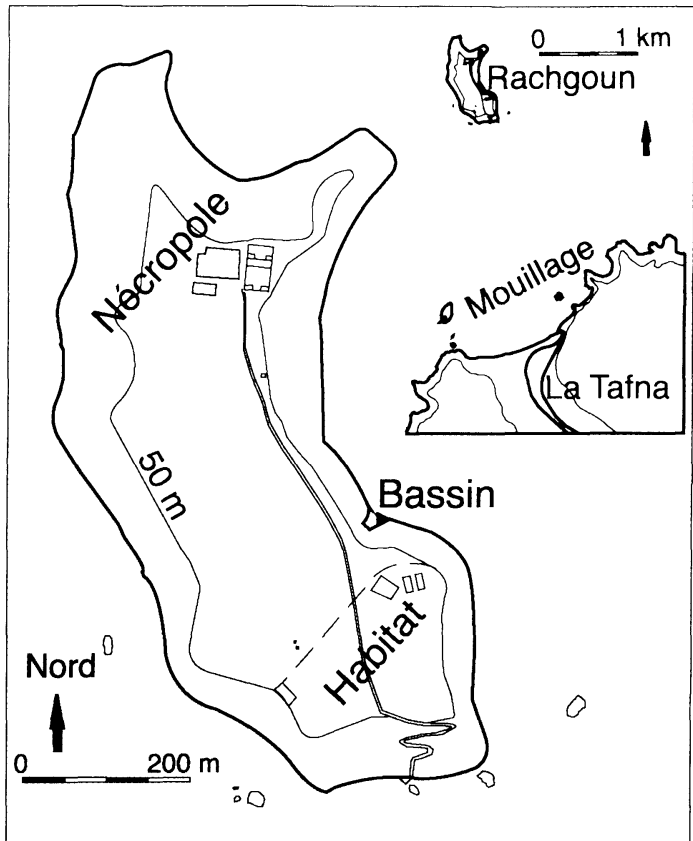

FIG. 8 - L'ÎLE DE RACHGOUN (d'après V(HILILMOT, 196.5)

Le cothon de Rachgoun est le plus modeste que l'on puisse rencontrer (fïg. 8). Une échancrure, large de $1,8 \mathrm{~m}$, taillée dans la falaise qui borde le côté est de l'île, constitue le chenal d'accès à un bassin rectangulaire de $20 \times 15$ m de côté, taillé dans le pouzzolane et dont le fond s'incline légèrement en direction du large (Vuillemot, 1965). Les dimensions réduites des aménagements rendent son utilisation comme port douteuse. Les fouilles de G. VuILLEMOT (1965) ont révélé une nécropole et un habitat de faible superficie dont le matériel daterait l'occupation de l'île de la deuxième moitié du VII s. et du VI"s. av. J.-C. (id.). Ce modeste bassin aurait alors constitué le seul abri où les barques pouvaient être échouées.

À Jezirat Fara'un (fïg. 9), le chenal d'accès au bassin est creusé sur environ $10 \mathrm{~m}$ avec une largeur de 2,5$3 \mathrm{~m}$. Le bassin apparaît aujourd hui comme vaguement rectangulaire, ses dimensions sont de $60 \times 30 \mathrm{~m}$ de côté, pour une profondeur de 2,5-3 $\mathrm{m}$. La paroi nord du bassin présente les vestiges d'un quai construit. Deux blocs identiques, perpendiculaires au rivage sud du bassin, et quelques blocs épars en périphérie laissent penser qu'il fut totalement aménagé (Flinder, 1977 ; RABAN, 1985). La datation des ces aménagements reste à préciser mais, d'après le matériel recueilli, il semble possible d'attribuer la première phase d'occupation de l'île, et de la création du port, au début du premier âge du Fer (Flinder, 1977). Cette datation renforcerait l'identification de Jezirat Fara'un avec Ezion Geber (id., 1986-87), la station navale établie au tout début du premier millénaire av. J.-C. par Salomon et Hiram Ir de Tyr, et mentionnée à plusieurs reprises dans la Bible (1 Rois 9, 26-27;10, 11-12;10, 22 ; 2 Chroniques 9, 10-11;9, 21).
FIG. 9 - LE COTHON DE JEZIRAT FARAUN (d'après FIINDER, 1977)

\section{Définition et typologie}

Au regard de cet inventaire succinct, il apparaît que la définition du cothon comme bassin portuaire creusé et débouchant sur la mer libre par l'intermédiaire d'un chenal, ne prend pas totalement en compte la diversité des vestiges archéologiques. En effet, le chenal et le bassin creusés ne sont que le dénominateur commun des exemples étudiés. La définition ne prend pas en compte les quais construits, les chenaux de désensablement, les hangars à navire, et d'autres aménagements, dont la fonction est quelquefois mal définie. De plus en ce qui concerne la technique mise en oeuvre, les vestiges archéologiques ne permettent d'attribuer le terme de «creusement» qu'à deux sites : Carthage et Mahdia.

À Carthage, les fouilles et les études géoarchéologiques (voir HURST, PASKOFF et RAKOB, 1985 ; Hurst, 1992 ; STAGER, 1992) ont établi qu'avant l'aménagement du port rectangulaire, puis du bassin circulaire, le secteur était une zone émergée plate, occupée par certains éléments de la ville archaïque, dont un chenal longeant le futur port rectangulaire et traversant l'îlot de l'Amirauté. Les deux bassins furent alors évidemment creusés dans le sol sablonneux (OuesLatı, 1993). À Mahdia, le bassin fut entièrement taillé dans le sol, constitué ici de grès calcaire pliocène (LEZINE,1965; PAskoff, Slim et Trousset, 1991). En revanche, à Motyé, Phalasarna, et Jezirat Fara'un, les travaux ne consistèrent pas à creuser un bassin, mais à aménager un plan d'eau déjà existant afin qu'il soit accessible à certains navires. À Phalasarna, le cothon fut établi dans une petite crique rocheuse par approfondissement d'un talweg, et élargissement de fissures naturelles mettant le bassin en relation avec la mer (HADJIDAKı, 1996). À Motyé, les Phéniciens auraient taillé le tuf argileux afin de régulariser les parois d'une dépression lagunaire (ISSERLIN, 1971 ; 1974 ; Mingazzinı, 1968). A Jezirat Fara’un, c'est une petite crique qui fut aménagée (FLINDER, 1977). 
Il apparaît alors clairement que deux techniques distinctes furent utilisées pour l’aménagement des cothons. ce qui nous a permis d'établir une typologie. La première technique (type A) consiste à creuser un bassin portuaire dans un terrain sec, et de le mettre en relation avec la mer par l'intermédiaire d'un chenal. L'avantage de cette technique est de donner une forme régulière au futur port, comme à Carthage et Mahdia où l'on observe des bassins de forme géométriques simples (rectangle ou cercle). La deuxième technique (type B) utilise les caractéristiques naturelles du site : des plans d'eau naturels, lagunes ou criques, sont aménagés de façon à constituer un port clos (Phalasarna, Motyé, Jezirat Fara'un). Plusieurs autres ports méditerranéens peuvent présenter une technique similaire. Il s'agit d'Amathonte à Chypre où un possible port intérieur, aujourd'hui comblé, pourrait avoir été établi dans une lagune (FroST, 1995 ; voir Aupert et al., 1979) ; de Kition, encore à Chypre, où le bassin du port hellénistique pourrait avoir été en partie artificiel (voir MORHANGe et al., 1999 ; 2000) ; de Lattaquié, en Syrie, où une petite crique pourrait avoir servi d'appui au port hellénistique (SAuvageT, 1934) ; et de Corinthe, où le bassin intérieur du Lechaion serait en partie creusé (SHAW, 1969). Tous ces exemples demandent ainsi à être étudiés selon une problématique spécifique qui viserait à déterminer avec précision le contexte géomorphologique précédent l'établissement du port.

\section{Vocation et utilisation}

On a considéré que l'aménagement d'un cothon était une technique simple et archaïque pour créer un port artificiel sur des côtes dépourvues d'abri naturel (BlaCKMAN, 1982 ; Frost, 1995). Deux observations contredisent cette suggestion. Tout d'abord, dans tous les cas signalés, hormis à Rachgoun dont l'accès à l'île est difficile, l'approche maritime du cothon est constituée par un lieu propice au mouillage; que ce soit dans une zone lagunaire comme à Motyé (fig. 7), au fond d'une baie protégée des vents et de la houle, comme à Jezirat Fara'un (fig. 9), Carthage (fig. 3) et, dans une moindre mesure, Phalasarna (fig. 6), ou sur le côté abrité d'un promontoire comme à Mahdia (fig. 5). Ensuite, le cothon n'est jamais l'unique port de l'établissement. À Jezirat Fara'un, il vient compléter un bon mouillage et un port continental. À Motyé, la quasi-totalité de la périphérie de l'île est propice à l'échouage des barques et deux autres ports sont construits à la même époque que le cothon ( $\mathrm{V}^{\mathrm{e}} \mathrm{s}$.) (voir Griffo Alabiso, 1991 ; Famà, 1995). À Carthage, ce sont le «quadrilatère de Falbe», le lac de Tunis, la baie du Kram ou la Marsa qui ont également fait office de port. À Rachgoun également, l'embouchure de la Tafna pourrait, dans l'antiquité, avoir constitué un port naturel (fig. 8). Le $Q$ apparaît alors plus comme complémentaire aux avantages portuaires naturels d'un site, que comme port majeur et unique. La mise en cuvre de tels chantiers nécessitant de gros efforts en hommes et en moyens, il semble logique de penser que ce type de bassin avait une vocation spécifique.
Si l'on s"attarde sur la situation urbaine des ports de type cothon, on constate que. excepté à Rachgoun, tous les sites sont situés à l’intérieur de l’enceinte de la ville. Cela ne fait aucun doute à Motyé où la muraille est même antérieure au cothon. À Carthage et à Phalasarna, si l'enceinte primitive de la cité ne semble pas englober la zone portuaire. des murs secondaires furent élevés tout autour. Les auteurs antiques ont bien noté cette caractéristique. À Carthage. Appien (Lib. 96) précise que la circulation à l'intérieur des ports, et entre les ports et la ville, était contrôlée par le franchissement de nombreuses portes. Lors de son passage à Phalasarna, le PseudoScylax ( $\$ 42$, éd. Müller) utilise l'expression de port «fermé» $(\kappa \lambda \varepsilon l \sigma \tau o ́ s)$. Comme l'a relevé K. LeHMANNHARTLEben (1923), cette dénomination signale un port intra-muros. Reste à préciser, dans les cas de Carthage et de Phalasarna, si les portions d'enceinte en relation avec les ports sont antérieures, contemporaines ou postérieures à l'aménagement du cothon. À Mahdia et à Jezirat Fara'un, les portions d'enceinte sont largement postérieures à l'aménagement du bassin. Peut-être reprennent-elle un tracé plus ancien ? Il apparaît tout de même légitime de supposer que la vocation principale du cothon était de créer un port à l'intérieur des murailles de la ville, conformément à la définition proposée par Sextus Pompeius Festus (op. cit.).

Reste à préciser l'utilisation d'un tel bassin. Le cas de Carthage est assez clair, le bassin circulaire, avec ses hangars à navires est uniquement utilisé par les militaires. Cela est largement attesté par le récit d'Appien (Lib. 96). Cependant, si une utilisation militaire du port peut justifier sa situation à l'intérieur de l'enceinte, qu'en est-il du port «marchand»? Il en va de même à Motyé, Rachgoun et Jezirat Fara'un où la faible superficie des bassins en interdisent l'accès aux navires de guerre. Le cothon serait, dans ces cas, un port à vocation commerciale. Dans ce sens, il est très probable que le choix d'une île à proximité du rivage comme agglomération portuaire répond à un souci de protection, tout comme la fortification de la totalité de la périphérie de l'île (Motyé et Jezirat Fara'un). Le golfe d'Aqaba, dans lequel est située l'île de Jezirat Fara'un, borde un désert où, dans l'Antiquité, la présence de pillards rendait le stockage des biens hasardeux. Il est probable que les caravanes en provenance de la Méditerranée déchargeaient sur le rivage leurs marchandises qui étaient ensuite transportées sur l'île grâce à de petites embarcations (RABAN, 1985). À Motyé, il est possible que la faible profondeur de la lagune ait interdit son accès aux gros navires, qui devaient mouiller en haute mer (FAMÀ, comm. pers.). Ici aussi de petites embarcations devaient assurer le transit entre la haute mer et la cité, sans doute par l'intermédiaire du cothon. Deux questions se posent alors : quelle était la particularité des marchandises en transit par le cothon de Motyé ? Pourquoi n'étaient-elles pas déchargées dans les autres ports de la ville ? Le même problème se pose à Carthage : quelle était la particularité des navires marchands qui pouvaient franchir l'enceinte de la ville, alors que d'autres utilisaient seulement le port extérieur ? 
Les vestiges archéologiques nous présentent ainsi le cothon comme un port totalement ou en partie artificiel. intra-muros, dont l'aménagement en arrière de la ligne de côte est complété par divers éléments à fonctions spécifiques (quais, hangars à navires. chenaux de désensablement...). Sa répartition chronologique et géographique traduit une technique privilégiée par les populations phéniciennes et puniques durant tout le premier millénaire avant notre ère, depuis Jezirat Fara'un (la base navale de Hiram I ${ }^{\mathrm{cr}}$ et de Salomon : Ezion Geber) au Premier âge du Fer, jusqu'à Carthage à la veille de sa destruction par les Romains ( 146 av. J.-C.), en passant par Motyé, Rachgoun et Mahdia. Dans le cas de Phalasarna, la relation avec les Phéniciens n'est pas établie. Nous mentionnerons que toute une série d'indices archéologiques traduit le développement d'une forte présence phénicienne en Crète, notamment à Cnossos (BAurain et Bonnet, 1992 ; Sznycer, 1979 ; FAlsone, 1987). À Phalasarna même, deux trônes d'Astarté suggèrent une présence phénicienne dans la ville (Frost 1995). Ces Phéniciens auraient-ils joué un rôle dans l'aménagement du cothon de Phalasarna ?

Quant à l'origine de la technique, il serait logique de se tourner vers la Phénicie, mais aucun exemple de cothon n'a été mis au jour dans cette région. Certes, Lattaquié, Kition et Amathonte sont voisins de la Phénicie, mais leurs ports ne sont pas définis comme cothon. Peutêtre doit-on alors s'aventurer encore plus à l'est, au-delà des rivages méditerranéens, sur les rives de l'Euphrate ? À Ur (fig. 10), à la fin du III ${ }^{\circ}$ millénaire, deux bassins étaient enclos dans l'enceinte de la ville, reliés par des canaux à l'Euphrate (BLACKMAN, 1982 ; WOOLLEY, 1930). À Til Barsib, au IX ${ }^{\mathrm{c}}-\mathrm{VIII} \mathrm{I}^{\mathrm{c}}$ s. av. J.-C., on trouve

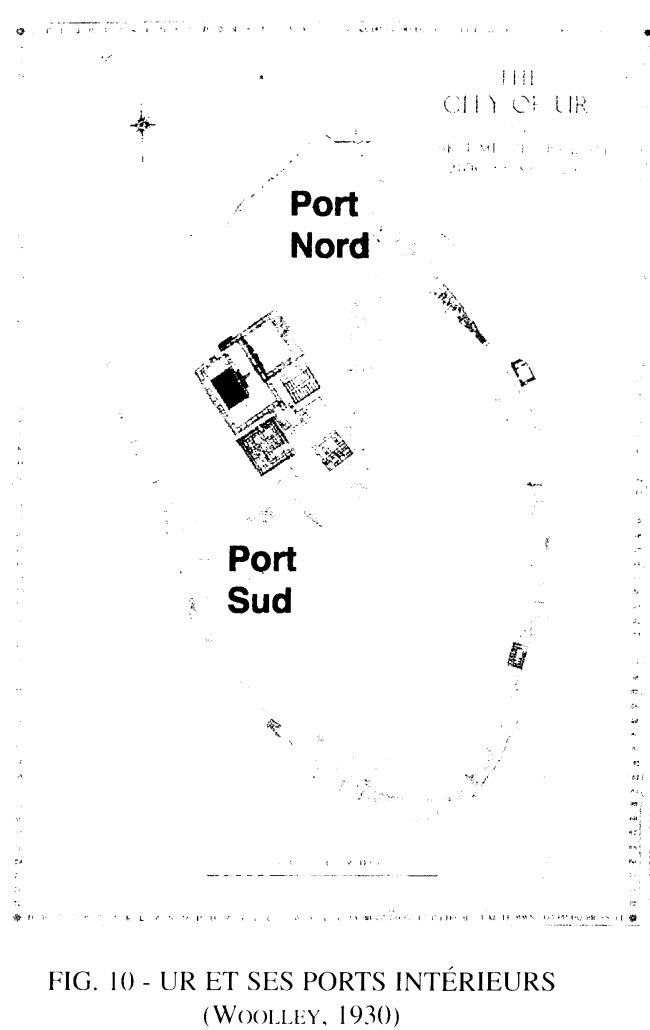

également un bassin taillé dans la berge du fleuve (Thureau-Dangin et Dunand, 1936). L'origine de la technique rejoindrait ainsi la thèse d'une origine sémitique au terme de cothon. Mais il serait auparavant nécessaire d'élargir la définition de Servius (op. cit.) : «Cothona sunt portus in mari...», et considérer que les ports de type cothon se trouvent aussi bien en contexte fluvial que maritime.

\section{BIBLIOGRAPHIE}

Aupert P., Hermary A., Tytgat C., Courtols L., Velde B., PARHAS C. et IANNIS S., (1979), Rapport sur les travaux de la mission française à Amathonte en 1978, $B C H$, vol.103, p. 725-763.

Baradez J., (1958), Nouvelles recherches sur les ports de Carthage, Karthago, vol. 9, p. 45-78.

Baurain C. et Bonnet C., (1992), Les Phéniciens : marins des trois continents, Armand Colin, Paris, $250 \mathrm{p}$

Ben Younes H., (1985), Rapport sur la campagne de fouilles effectuée dans la grande nécropole de la région de Mahdia, octobre-novembre 1982, Revue des Etudes PhéniciennesPuniques et des Antiquités Libyques, vol. 1, p. 23-61.

Blackman D. J., (1982), Ancient Harbours in the Mediterranean, Part 1, International Journal of Nautical Archaeology, vol. $11, \mathrm{n}^{\circ} 2$, p. $79-104$

Daremberg C. et Saglio E., éds., (1962-63), Dictionnaire des Antiquités Grecques et Romaines d'après les textes et les documents, Akademische Druck, Graz, 10 vol., impression photomécanique de l'édition Hachette, Paris, 1877-1919.

Debergh J. et LiPINSKI E., (1992), s.v. «cothon», in LIPINSKI E. (dir.), Dictionnaire de la civilisation phénicienne et punique. Brepols, p. 121.

Du Plat Taylor J., (1964), Motya, a Phoenician Settlement in
Sicily, Archaeology, vol. 17, n ${ }^{\circ}$, p. 91-100.

FAlsone G., (1987), La coupe phénicienne de Fortetsa, Crète : une reconsidération, Studia Phoenicia, vol. V, p. 181-194.

FAMÀ M.L., (1995), Il porto di Mozia, Sicilia Archeologica, vol. 28, p. 171-180.

FAnTAR M.H., (1999), Villes-ports de Byzacène avant la conquête romaine, in La Méditerranée : l'homme et la mer, dans le cadre du Projet National Mobilisateur: Les villesports en Tunisie, Cahiers du CERES, série Géographique, $\mathrm{n}^{\circ} 21$, Tunis, p. 13-51.

Flinder A., (1977), The Island of Jezirat Faraun, its ancient harbour, anchorage and marine defence installations, International Journal of Nautical Archaeology, vol. 6, $\mathrm{n}^{\circ}$ 2, p. 127-139.

Fuinder A., (1986-87)., The Search for Ezion-Geber, Bulletin of the Anglo-Israel Archaeological Society, vol. 6, p. 43-45.

FoUCHER L., (1964), Hadrumetum, Presses Universitaires, Tunis, $405 \mathrm{p}$.

Frost F.J., (1997). Tectonics and History at Phalasarna, in Swiny S., Hohlfelder R.L., et Wylde SWiny H. (éds.), Res Maritimae, American School of Oriental Research, Atlanta, p. 107-115.

Frost H., (1995). Harbours and proto-harbours ; early levantine 
engineering. in KaRatitorghHS V. et MICHAELIIDES D (éds.). Cyprus and the Sea proceedings of the International Simposium. Nicosia 25-26/l0//99.3. p.1-22

Griflo) Al.abiso M.G.. (1991). La Strada di Mozia nello Stagnone di Marsala. Sicilia Archeologica. vol. 24. p. $77-80$

Gsta... S.. (1928). Histoire Ancienne de l'Africue du Nord. tome II. Hachette. Paris, 475 p.

HADJIDAKI E. (1988). Preliminary Report of the Excavations at the Harbor of Phalasarna in West Crete. American Journal of Archaeology, vol. 92. p. 463-479.

HADJIDAKI E., (1996), The Hellenistic Harbor of Phalasarna in Western Crete : A Comparison with the Hellenistic Inner Harbour of Straton's Tower, in RaBAN A. et Holum K.G. (éds.), Caesarea Maritima : a Retrospective After Two Millenia, Hardcover, p. 53-64.

Hurst H., (1975-80), Excavations at Carthage, 1974-1978, FirstFourth Interim Report. Antiquaries Journal, vol. 55, p.1139; vol. 56, p.177-197; vol. 57, p.232-261; vol. 59, p.1949.

Hurst H., (1992), L'îlot de l'Amirauté, le port circulaire et l'avenue Bourguiba, in Ennabli A. (dir.), Pour samver Carthage, exploration et conservation de la cité punique, romaine et byantine, UNESCO/INAA, Paris-Tunis, p.7994.

Hurst H., (1993), Le port militaire de Carthage, Les dossiers d'Archéologie, vol. 183, p. 42-51.

Hurst H., Paskoff R. et Rakob F., (1985), Géologie maritime : position du niveau de la mer et déplacement de la ligne de rivage à Carthage (Tunisie) dans l'Antiquité. Comptes rendus de l'Académie des Sciences, 2' série, vol. 300, $\mathrm{n}^{\circ} 13$, p. 613-618.

Hurst H. et Stager L.E., (1978), A Metropolitan Landscape : The Late Punic Port of Carthage, World Archaeology. vol. $9, \mathrm{n}^{\circ} 3$, p. 334-346.

ISSERLIN B.S.J., (1971), New Light on the «cothon» at Motya, Antiquity, vol. 45, p. 178-186, pl. XXVII-XXX.

ISSERLIN B.S.J., (1974), The Cothon at Motya: Phoenician harbour works, Archaeology, vol. 27, nº 3, p. 188-194.

Lehmann-Hartleben K., (1923), Die Antiken Hafenanlagen des Mittelmeeres, Beiträge zur Geschichte des Städtbaues im Altertum, Klio XIV, Dieterich'sche Verlagsbuch handlung, Leipzig, $304 \mathrm{p}$.

LÉzINE A., (1965), Mahdiva, recherche d'archéologie islamique, Klincksieck, Paris, $148 \mathrm{p}$.

MingazZinı P., (1968), Scopo e natura del cosidetto kothon di Mozia, in Mozia - IV. Rapporto preliminare della Missione archeologica della Soprintendenza alle Antichità della Sicilia Occidentale e dell'Università di Roma, Università di Roma, Studi Semitici 29, Roma, p. 105-112.

Morhange C., Goiran J.-P., Bourcier M., Carbonel P., Le Campion J., Pyatt B., Prone A., Rouchy J.-M., SOURISSEAU J.-C. et YoN M., (1999), 3000 ans de modifications des environnements littoraux à Kition Bamboula. Larnaca. Chypre. Méditerranée Orientale. Quaternaire. vol. 2-3. p. 133-149.

Morhangh: C.. Golran J.-P., Bolrcifer M., Carbonel. P., Lh

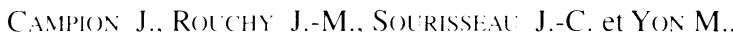
(20)(0). Recent Holocene paleo-environmental evolution and coastline changes of Kition. Larnaca. Cyprus. Mediterranean Sea. Marine Geology, vol. 170). p. 205-230.

Ouestati A. (1993). Lés côtes de la Tunisie. Facultés des Sciences Humaines et Sociales. Tunis, 2 vol., 387 et 402 p.

Paskoli: R.. Silm H. et Trousset P., (1991). Le littoral de la Tunisie dans l'Antiquité : cing ans de recherches géoarchéologiques, Comptes Rendus de l'Académie des Inscriptions et Belles-Lettres, p. 515-546.

Pirazzol. P.A., Ausseill-Badie J., Giresse P., Hadjidaki E. et ARNOL.D M., (1982). Historical Environmental Changes at Phalasarna Harbour, West Crete, Geoarchaeology, vol. 7 , $\mathrm{n}^{\circ} 4$, p. $371-392$

RABAN A., (1985), The Ancient harbour of Israel in Biblical Times (From the Neolithic Period to the End of the Iron Age), in Raban A. (éd.), Harbour Archaeology. Proceedings of the first international workshop on ancient Mediterranean harbours. Caesarea Maritima, 24-28.6.83, BAR International Series 257, Haifa, p. 11-40.

Sauvaget J., (1934), Le plan de Laodicée-sur-Mer, Bulletin des Etudes Orientales, vol. 4, p. 81-114.

SHAw J., (1969), A Foundation at the Inner Harbor at Lechaum, American Journal of Archaeology, vol. 73, p. 370-372.

Spanò Giammelario A., (1997), Les Phéniciens et les Puniques en Sicile, Les Dossiers d'Archéologie, vol. 225, p. 22-32.

Stager L.E., (1992), Le tophet et le port commercial, in EnNABLI A. (dir.), Pour sauver Carthage, exploration et conservation de la cité punique, romaine et byzantine, UNESCO/INAA, Paris-Tunis, p. 73-78.

SZNYCER M., (1979), L'inscription phénicienne de Tekke, près de Cnossos, Kadmos 18 (1979), p.89-93.

Thureau-Dangin F. et Dunand M., (1936), Til Barsib, P. Geuthner, Paris, 167 p.

Viedebantt, O., (1922), s.v. Kothon, in Pauly H. F. von, Wissowa G., (éds), Paulys Real-Encyclopädie der Classischen Altertumswissenschaft, J.B. Metzler, Stuttgart-München, vol. 11, col. p.1516-1520.

Vuillemot G., (1965), Reconnaissances aux échelles puniques d'Oranie, Musée Rollin, Autin, 454 p.

Whitaker J.I.S., (1921), Motya: a Phoenician Colony in Sicily, G. Bells \& Sons, London, 357 p.

Woolley C.L., (1930), Excavations at Ur, 1929-1930, Antiquaries Joumal vol. 10, $\mathrm{n}^{\circ}$ 4, p. 315-343.

Zaoual. L., (1999), Mahdia, port et arsenal, in La Méditerranée : I'homme et la mer, dans le cadre du Projet National Mobilisateur : "Les villes-ports en Tunisie», Cahiers du CERES, série Géographique, $\mathrm{n}^{\circ}$ 21, Tunis, p.219-239.p 